\title{
ANÁLISE DO COEFICIENTE DE MORTALIDADE MATERNA NO MUNICIPIO DE OSASCO, S. PAULO, BRASIL *
}

\section{RSPSP-136}

Ciari JR., C. \& Almeida, P.A.M. de - Análise do coeficiente de mortalidade ma. terna no município de Osasco, S. Paulo, Brasil. Rev. Saúde públ., S. Paulo, 6: $237-44,1972$.

REsumo: Foi analisado o coeficiente de mortalidade materna no municipio de Osasco e observado que o mesmo ainda é alto comparado ao de S. Paulo (Capital) $e$ ao de cidades localizadas em paises mais desenvolvidos. Porém, o comportamento deste coeficiente, nos diversos anos, mostra efetiva melhora nos serviços de saúde materna, através da redu. ção de 28,99\% no período de 1967/1970. Observou-se que, ao ser analisado o coeficiente de mortalidade materna deve ser levado em consideração não só a sua magnitude, como também o seu comportamento nos diferentes anos. Além disso, deve-se considerar a proporção que apresenta em relação às demais causas de óbitos no grupo fértil feminino e o relacionamento entre si das causas obstétricas. Foi salientada a correspondência existente entre causas obstétricas de óbito $e$ serviços de saúde materna.

UnITERMos - Saude materna*; Mortalidade materna*; Assistência pré-natal* .

IN TRODU C $\overrightarrow{\mathrm{A} O}$

$O$ coeficiente de mortalidade materna, expressado pelo número de óbitos ocorridos por causas de gravidez, parto, puerpério ou aborto sobre nascidos vivos por
Cyro CIARI Jr.

Pedro Augusto Marcondes de ALMEIDA

1.000 , tem sofrido algumas críticas. No entanto, representa ainda a melhor forma de se medir a intensidade do evento, sendo sistematicamente usado.

A ocorrência de obito materno tem diminuído sensivelmente em países onde se desenvolvem intensos programas de Saúde Materna. Isto ocorre, por exemplo, na cidade de São Francisco, onde o coeficiente é de 1,1/10.000, o mesmo aconte. cendo na cidade de Bristol (1,8/10.000) 4 . Porém, em áreas menos desenvolvidas, a frequiência do evento óbito por causas obstétricas ainoa se mantem alta, como: Bogotá, 12.6; Lima, 15.6; Santiago, 31.6/10.000 habitantes.

Na análise da mortalidade materna, para que seja adequadamente compreendida e para que expresse os fatores que a estão determinando, devemos levar em consideração alguns aspectos básicos:

1 - Magnitude do coeficiente comparado com outras áreas;

2 - comportamento do coeficiente nos diversos anos;

3 - proporção dos óbitos maternos em relação a outras causas de óbito de mulheres em idade fértil;

4 - proporção das causas de óbito materno entre si;

* Da Disciplina de Saúde Materna do Departamento de Prática de Saúde Pública da USP Av. Dr. Analdo, 715 - São Paulo, S. P., Brasil. 
CIARI Jr., C. \& ALMEIDA, P. A. M. de - Análise do coeficiente de mortalidade materna no município de Osasco, S. Paulo, Brasil. Rev. Sande públ., S. Paulo, 6: 237.44, 1972.

\section{5 - proporção de óbitos maternos ocorridos durante a gravidez, o parto, o puerpério e por aborto.}

Desta forma, não basta sabermos a magnitude do coeficiente de mortalidade materna. É necessário interpretá-lo em seus aspectos mais intimos. $O$ seu comportamento durante os anos observados, nos dá a medida da atuação de serviços de maternidade. Além disso, devemos conhecer a sua situação em relação ao número de óbitos que ocorrem no grupo fértil feminino pois, naturalmente, deve sempre estar em último lugar, dado ser a gravidez um processo fisiológico pouco determinante de óbito. Porém, este enfoque deve ainda ser estendido ao comportamento das causas obstétricas entre si, pois, cada uma delas expressa com sua presença, uma deficiência de serviços. De uma maneira geral, os óbitos ocorridos por toxemia, hemorragia ou infecção mostram deficiência, seja na assistência pré-natal, ou seja, na assistência ao parto. Na avaliação dos serviços de saúde materna ainda é importante conhecer-se a proporção dos óbitos que estão ocorrendo na gravidez, no parto ou no puer. pério que mais uma vez evidenciam as deficiências de serviços.

Pretendemos analisar a situação da mortalidade materna no município de Osasco levando em consideração estes 5 aspectos básicos. Desta forma, poderemos aquilatar a situação da saúde materna naquele município enfocada apenas pelo aspecto de perdas de gestantes.

O pequeno número de óbitos, como é usual em saúde materna, não nos permite, pela análise destes dados, extrapolar para outras áreas ou afirmar um comportamento uniforme dos eventos. Contudo, representa subsídio para uma observação mais prolongada da ocorrência que permitirá então uma conclusão segura. Da mesma forma, deixamos de fazer a análise de óbitos maternos por aborto, dada à escassez de dados. No entanto, fo- ram incluídos para o cálculo do coeficiente de mortalidade materna.

\section{MATERIAL E METODOS}

Foram levantados no Cartório de Registro Civil de Osasco, os óbitos ocorridos nos anos de 1967 a 1970, inclusive. Destes, foram retirados os femininos que ocorreram na idade de 15-50 anos. Do montante destes óbitos tomamos aqueles onde havia a indicação de causa obstétrica. Nestes procurava_se classificar as causas do obito e sempre que necessário recorreu-se ao médico assistente ou registro do hospital para esclarecimento, principalmente para identificar a idade da gestação e se o óbito tinha ocorrido na gestação, no parto ou no puerpério. $\mathrm{Na}$ classificaçäo de causas adotaram-se os critérios da Classificação Internacional de Doenças, revisão de $1965^{2}$. Quando dividimos os óbitos maternos ocorridos durante a gravidez, o parto e o puerpério, adotamos o critério de situá--los de acordo com a causa básica do óbito. Assim, um caso de infecção puerperal estará situado no puerpério, porém, se a infecção iniciou-se no parto (infecção intra-parto) foi classificado neste grupo e não mais em puerpério.

Não levamos em consideração a evasão de partos que ocorre no município dada sua proximidade de São Paulo. Acreditamos que os números obtidos em Osasco exprimem a realidade local, isto é, o nú. mero de partos que são assistidos no município e para os quais são requeridos serviços.

A população foi estimada consideranse os saldos migratório e vegetativo achados mediante coeficientes médios dos censos, extrapolando linearmente. Nesta estimativa, por este metodo, achamos erro de $2,93 \%$ para mais em relação ao censo de 19701.

Para comparar a proporção de óbitos por causas maternas e as demais causas 
CIARI Jr., C. \& ALMEIDA, P. A. M. de - Análise do coeficiente de mortalidade materna no município de Osasco, S. Paulo, Brasil. Rev. Saúde públ., S. Paulo, 6: 237-44, 1972.

de morte em mulheres na idade fértil, escolhemos algumas mais significantes e grupamos outras. Ao lado das causas maternas colocamos neoplasias malígnas, moléstias renais mais hipertensão, cardiopatias e, num único conjunto, as demais.

O grupo feminino em idade reprodutiva consideramos como compreendido entre 15-50 anos. Alguns autores como Puffer
\& GRIFFITH ${ }^{5}$ preferem 15-45. No entanto, o escolhido é mais frequientemente usado em nossa literatura.

\section{RESULTADOS}

A magnitude dos coeficientes de mortalidade materna no município de Osasco e sua comparação com a Capital, interior e o Estado são vistos na Tabela 1.

TA BE A 1

Coeficientes da mortalidade materna no Municipio de Osasco, na Capital e Interior do Estado 1965/70

\begin{tabular}{l|c|c|c|c|c|c}
\hline & \multicolumn{7}{c}{ Coeficientes por 1.000 nascidos vivos } \\
\cline { 2 - 7 } & 1965 & 1966 & 1967 & 1968 & 1969 & 1970 \\
\hline Capital & 0,87 & 0,98 & 0,78 & 0,73 & - & - \\
Interior & 1,34 & 1,34 & 1,37 & - & - & - \\
Estado & 1,20 & 1,23 & 1,19 & - & 1,17 & 1,20 \\
Osasco & - & - & 1,69 & 1,42 & - \\
\hline
\end{tabular}

Fonte: Instituto de Saúde ${ }^{3}$

Departamento de Estatística do Estado de são Paulo *

Secretaria de Saúde de Osasco 1

Verificamos que nos anos em que é possivel a coincidência de dados, Osasco mantinha seu coeficiente mais alto que os demais. No entanto, verifica-se que a tendência de Osasco e Capital é para redução progressiva dos coeficientes, ao passo que no Interior permanecem estáveis.

A proporção de óbitos maternos relacionados com outras causas de óbito de mulheres em idade fértil, no municipio de Osasco, é vista na Tabela 2.

Pela Tabela 2, nota-se que os óbitos por causa obstétrica são sempre os de me- nor proporção, seguindo-se carđiopatias, moléstias renais e câncer. O grupo de outras causas naturalmente supera as demais.

Durante os anos observados, verifica se ainda que a proporção de obitos obstétricos em relação aos demais vem se mantendo dentro de, aproximadamente, um décimo do total de mortes do grupo.

Interessante ainda é verificar a proporção de óbitos por idade dentro do grupo fértil feminino. Para isto somamos as mortes ocorridas nos 4 anos e distribufmos por idade e causas situando sua proporção. (Tabela 3). 
CTARI Jr., C. \& ALMFIDA, P. A. M. de - Análise do coeficiente de mortaliaade materna no municipio de Osaseo, S. Paulo, Brasil. Rev. Saúde públ., S. Paulo, 6: 237-44, 1972.

\section{TABEA 3}

Proporção de óbitos por causas obstétricas relacionadas às causas de câncer, moléstias renais e hipertensivas, cardiopatias e outras causas no grupo feminino fértil (15 - 50 anos)

\begin{tabular}{|c|c|c|c|c|c|c|c|c|c|c|c|}
\hline \multirow[b]{2}{*}{ Ano } & \multirow{2}{*}{ Total } & \multicolumn{2}{|c|}{$\begin{array}{c}\text { Obstétrica } \\
\text { (B.41) }\end{array}$} & \multicolumn{2}{|c|}{$\begin{array}{r}\text { Câncer } \\
\text { (B.19) }\end{array}$} & \multicolumn{2}{|c|}{$\begin{array}{c}\text { Renais e } \\
\text { Hipertensivas } \\
\text { (B.27-B.38) }\end{array}$} & \multicolumn{2}{|c|}{$\begin{array}{l}\text { Cardiopatias } \\
\text { (B26-B28_B29) }\end{array}$} & \multicolumn{2}{|c|}{$\begin{array}{l}\text { Outras } \\
\text { Causas }\end{array}$} \\
\hline & & N. ${ }^{\circ}$ & $\%$ & N. ${ }^{\circ}$ & $\%$ & N..$^{\circ}$ & $\%$ & N.o & $\%$ & N: & $\%$ \\
\hline 1967 & $79(100 \%)$ & 9 & 11,39 & 5 & $6, \overline{5} 0$ & 18 & 22,78 & 23 & 29,11 & 24 & 30,37 \\
\hline 1968 & $92(100 \%)$ & 8 & 3,69 & 12 & 13,04 & 23 & 25,00 & 28 & 30,43 & 21 & 22,82 \\
\hline 1969 & $82(100 \%)$ & 7 & 8,53 & 10 & 12,19 & 15 & 18,29 & 23 & 28,04 & 27 & 32,92 \\
\hline 1970 & $76(100 \%)$ & 8 & 10,52 & 13 & 17,10 & 19 & 25,90 & 15 & 19,73 & 21 & 27,63 \\
\hline
\end{tabular}

Fonte: Secretaria de Saúde de Osasco 1

* Os números entre parêntesis referem-se àqueles da lista abreviada (B) da 8.a Revisão (1965) da Classificação Internacional de Doenças ${ }^{2}$.

T A B E L A 3

Número e porcentagem de óbitos no grupo feminino fértil (15 - 50) ocorrido no Municíplo de Osasco de 1967 a 1970

\begin{tabular}{|c|c|c|c|c|c|c|c|c|c|c|c|c|}
\hline \multirow[b]{2}{*}{$\begin{array}{l}\text { Grupo } \\
\text { Etário }\end{array}$} & \multicolumn{2}{|c|}{ Total } & \multicolumn{2}{|c|}{$\begin{array}{c}\text { Obstétrica } \\
\text { (B.41) }\end{array}$} & \multicolumn{2}{|c|}{$\begin{array}{c}\text { Cancer } \\
\text { (B.19) }\end{array}$} & \multicolumn{2}{|c|}{$\begin{array}{c}\text { Renais e } \\
\text { Hipertensivas } \\
\text { (B.27-B.38) }\end{array}$} & \multicolumn{2}{|c|}{$\begin{array}{l}\text { Cardiopatias } \\
\text { (B26-B28_B29) }\end{array}$} & \multicolumn{2}{|c|}{$\begin{array}{l}\text { Outras } \\
\text { Causas }\end{array}$} \\
\hline & N.o & $\%$ & N:O & $\%$ & N.o & $\%$ & N.o & $\%$ & N. ${ }^{\circ}$ & $\%$ & N.o & $\%$ \\
\hline $15-20$ & 14 & 4,29 & 2 & 14,28 & 0 & - & 1 & 7,14 & 4 & 28,58 & 7 & 50,00 \\
\hline $20-25$ & 18 & 5,52 & 4 & 22,22 & 0 & - & 1 & 5,55 & 2 & 11,11 & 11 & 61,11 \\
\hline $25-30$ & 46 & 14,11 & 5 & 100,89 & 2 & 4,34 & 8 & 17,39 & 11 & 23,91 & 20 & 43,47 \\
\hline $30-35$ & $4 i j$ & 13,80 & 6 & 13,33 & 4 & 8,88 & 7 & 15,55 & 16 & 35,55 & 12 & 26,66 \\
\hline $35-40$ & 57 & 17,48 & 7 & 12,28 & 5 & 8,77 & 12 & $21 ; 05$ & 21 & 36,84 & 12 & 21,05 \\
\hline $40-45$ & 65 & 19,93 & 8 & 12,30 & 11 & 16,92 & 19 & 29,23 & 17 & 26,15 & 10 & 15,38 \\
\hline $45-50$ & 81 & 24,84 & 0 & - & 18 & 22,22 & 24 & 29,62 & 18 & 22,22 & 21 & 25,92 \\
\hline Total & 326 & 100,00 & 32 & 9,81 & 50 & 15,33 & 72 & 22,08 & 89 & 27,20 & 93 & 28,52 \\
\hline
\end{tabular}

Fonte: Secretaria de Saúde de Osasco ${ }^{1}$

* Os números entre parêntesis referem-se àqueles da lista abreviada (E) da 8.* Revisão (1965) da Classificação Internacional de Doenças ${ }^{2}$

Verificamos que a distribuição dos obitos se faz de acordo com a idade mais comum de incidência das moléstias.
Outro aspecto a destacar é a proporção mantida pelos óbitos de causa obstétrica entre si. (Tabela 4). 
CIARI Jr., C. \& ALMEIDA, P. A. M. de - Análise do coeficiente de mortalidade materna no municipio de Osasco, S. Paulo, Brasil. Rev. Saúde publ., S. Paulo, 6: 237-44, 1972.

T A B E L A 4

Proporção de óbitos por causas obstétricas (Hemorragia, Toxemia, Infecção) no Município de Osasco nos anos de 1967 a 1970.

\begin{tabular}{|c|c|c|c|c|c|c|c|}
\hline \multirow[b]{2}{*}{ Ano } & \multirow{2}{*}{ Total } & \multicolumn{2}{|c|}{ Hemorragia } & \multicolumn{2}{|c|}{ Toxemia } & \multicolumn{2}{|c|}{ Infecção } \\
\hline & & N.o & $\%$ & N.o & $\%$ & N.o & Yo \\
\hline 1967 & 9 & 3 & 33,33 & 3 & 38,33 & 3 & 33,33 \\
\hline 1968 & 8 & 4 & 50,00 & 3 & 37,50 & 1 & 12,50 \\
\hline 1969 & 7 & 1 & 14,28 & 5 & 71,42 & 1 & 14,28 \\
\hline 1970 & 8 & 3 & 37,50 & 4 & 50,00 & 1 & 12,60 \\
\hline Total & 32 & 11 & 34,37 & 15 & 46,87 & 6 & 18,75 \\
\hline
\end{tabular}

Fonte: Secretaria de Saúde de Osasco ${ }^{1}$.

Vemos que a toxemia se situa em primeiro lugar, seguida de hemorragia e infeç̧ão, distribuição esta característica do nosso meio.
Finalmente devemos verificar o comportamento dos 6 bitos em relação a sua ocorrência na gestação, no parto e no puerpério.

T A B E L A 5

Proporção dos óbitos de causa obstétrica ocorridos na gravidez, no parto e no puerpério, no Municíplo de Osasco do ano de 1967 a 1970.

\begin{tabular}{l|c|c|c|c|c|c|c}
\hline \multirow{2}{*}{ Causa } & \multirow{2}{*}{ Total } & \multicolumn{2}{|c|}{ Gravidiez } & \multicolumn{2}{|c|}{ Parto } & \multicolumn{2}{c}{ Puerpério } \\
\cline { 3 - 8 } & & N.0 & $\%$ & N.0 & $\%$ & N.0 & $\%$ \\
\hline 1967 & 9 & 3 & 33,33 & 4 & 44,44 & 2 & 22,22 \\
1968 & 8 & 4 & 50,00 & 2 & 25,00 & 2 & 25,00 \\
1969 & 7 & 4 & 57,14 & 2 & 28,57 & 1 & 14,28 \\
1970 & 8 & 3 & 37,50 & 3 & 37,50 & 2 & 25,00 \\
\hline Total & 32 & 14 & 43,75 & 11 & 34,37 & 7 & 21,87 \\
\hline
\end{tabular}

Fonte: Secretaria de Saúde de Osasco ${ }^{1}$.

Observa-se uma predominância para eventos ocorridos na gestação e em menor porcentagem no puerpério 
CIARI Jr., C. \& ALMEIDA, P. A. M. de - Análise do coeficiente de mortalidade materna no município de Osasco, S. Paulo, Brasil. Rev. Saúde públ., S. Paulo, 6: 237-44, 1972.

\section{COMENTARIOS}

Como já tinhamos assinalado o coeficiente de mortalidade materna mede 0 número de óbitos que estão ocorrendo em relação aos nascidos vivos. Sendo medida quantitativa este valor aparece principalmente quando são comparadas áreas diferentes.

$\mathrm{Na}$ análise do coeficiente de Osasco, comparando-se com São Paulo, Capital, observa-se considerável diferença que já não é tão acentuada quando comparada com o Estado e é menor em relação ao interior. No entanto, se observarmos o comportamento do coeficiente de Osasco em relação aos demais da Tabela 1 , vemos que para a Capital, do ano de 65 para o de 68 , houve uma redução de $16,09 \%$, ao passo que em Osasco a redução foi de $28,99 \%$, no período de $67 / 70$.

Desta forma parece que a tendência dos serviços de Saúde Materna no Muni. cípio de Osasco é no sentido de atuar de forma significativa na redução da mortalidade materna.

A Tabela 2, demonstra que as causas obstétricas situam-se abaixo das demais causas escolhidas para comparação neste trabalho. No grupo fértil feminino esta deve ser a situação normal do ítem, causas obstétricas. Por outro lado mantem durante anos a proporção de $1 / 10$ das restantes causas.

Importante de se considerar para análise dos serviços de assistência à maternidade é a situação do porcentual de óbitos por causas obstétricas em relação às demais causas. Observamos sob este aspecto que o porcentual de obitos por cardiopatia suplanta em muito o de causas obstétricas, assim como o de renais e hipertensão arterial que se segue em importância ao grupo de cardiopatias. $O$ porcentual devido aos tumores malignos segue-se ao de renais e hipertensão arterial, apresentando também índice mais elevado do que o de causas obstétricas.
Puffer \& Griffith 5 em seu trabalho sobre o grupo etário feminino de 15-74 anos, no período de 1962 a 1964, observaram praticamente o mesmo em relação a várias cidades. Especialmente sobre São Paulo e Ribeirão Preto, encontraram como coeficiente mais elevado de causa d3 óbito, em primeiro lugar, as cardiopatias, seguindo-se os tumores malígnos.

Desta forma, o grupo fértil feminino do Município de Osasco está se mantendo, no que diz respeito aos óbitos de causa obstétrica, em nível porcentual semelhante ao existente em outras cidades.

Dados importantes são colhidos na Ta. bela 3 , onde relacionamos as diversas causas de óbito com vários grupos etários. Observamos que no referente ao total de óbitos há uma elevação da porcentagem de forma diretamente proporcional à elevação da idade dos grupos etários. O mesmo já não podemos constatar em relação aos óbitos maternos, pois estes se mantêm praticamente estáveis em todos os grupos etários, caindo a 0 no grupo etário 45-50 anos. Quanto aos óbitos por câncer, a tabela reflete evidentemente o que já se esperava, isto é, a elevação rápida e efetiva do porcentual a partir do grupo etário 25-30, atingindo o máximo no grupo de 45-50. O mesmo observamos no que diz respeito aos óbitos por hipertensão arterial e renais, sendo que já no grupo etário mínimo, 15-20 anos, são encontrados.

As cardiopatias atuam sobre todos os grupos etários indiferentemente (Tabela 3). As outras causas de óbitos, por reunirem um grupo muito heterogêneo de moléstias, atuam diferentemente, atingindo porcentual elevado nos grupos etários baixos e diminuindo a medida que se eleva a idade das pacientes.

Um dos aspectos importantes da mortalidade materna é verificar a porcentagem com que participam as diferentes causas obstétricas. $\mathrm{Na}$ dependência dos 
CIARI Jr,, C. \& ALMEIDA, P. A. M. de - Análise do coeficiente de mortalidade materna no município de Osasco, S. Paulo, Brasil. Rev. Saúde públ., S. Paulo, 6: 237-44, 1972.

Serviços de Assistência Materna oferecidos, varia a importância de cada uma delas. A oferta de antibióticos relegou a plano secundário o bbito por infecção, da mesmo forma que o uso adequado do sangue permite controlar o obito por hemorragia . A toxemia, moléstia própria da gestação, por suas características, é controlada pelos serviços de pré-natal. Desta forma a maior ou menor incidência de uma destas causas está relacionada ao tipo de atividade desenvolvida no programa de Saúde Materna. Assim, as deficiências de serviços de pré-natal colocam a toxemia em primeiro lugar, bem como as dificuldades na assistênca ao parto incrementam os óbitos por hemorragia $e$ infeç̧ão.

Desta forma, um serviço adequado de assistência à maternidade deve determinar a seguinte ordem cronológica de importância: toxemia, hemorragia e infecção. Os óbitos por aborto devem merecer análise especial dada as dificuldades que cercam seu registro. Outra variável, não introduzida neste trabalho, por falta de dados precisos, é a dos óbitos por causas associadas à gravidez, como cardiopatias, hemopatias e outras que, na verdade, deveriam ser as mais importantes e que não aparecem pela falta de registros precisos, perdendo-se entre as causas de óbitos gerais.

A Tabela 4, mostra a situação de Osasco onde vemos a predominância da toxemia $(46,87)$ seguida por hemorragia $(34,37)$ e infecção $(18,75)$. No entanto, vemos que em 1967 a importância das três se equivalia, ao passo que em 1968 a hemorragia predominou e só houve ajustamento a partir de 1969, necessitando maior observação para verificar se estes resultados se mantêm. Isto revela que é muito recente a melhoria de serviços da área de Saúde Materna. Nesta nova linha de idéias a situação dos óbitos ocorridos durante a gestação, o parto e o puerpério também dá uma visão do estado da assistência no local. Na Tabela 5, encontramos a porcentagem maior durante a gravidez, seguida de parto e puerpério. Estes dados coincidem com os de 6bitos, na Tabela 4 .

Estes resultados da análise dos fatores que interferem com o coeficiente de mortalidade materna, demonstram uma coe rência entre os níveis de ređução da mortalidade e o comportamento intrínseco da redução, pois, se bem que devamos considerar o coeficiente como alto, ele está se reduzindo progressivamente e as causas de óbito materno estão ocupando uma proporção correta.

\section{CONCLUS OES}

1 -O coeficiente de mortalidade materna no Município de Osasco é alto comparado ao de São Paulo (Capital) e ao de cidades de países mais desenvolvidos.

2-O importante na análise do coefi ciente de mortalidade materna é o seu comportamento nos diversos anos, como indicativo de melhoria de serviços de maternidade.

3-A relação entre os óbitos por causas obstétricas com as demais no grupo fértil feminino, demonstra que as de gravidez, parto e puerpério ocuparam o último lugar en tre todas as causas.

4-A proporção entre os óbitos de causa obstétrica presta-se para avaliar não só a efíciência dos serviços de assistência à maternidade, como também identificar suas deficiências relacionadas às atividades de pré-natal e assistência ao parto. 
CiARI Jr., C. \& ALMmida, P. A. M. de - Análise do coeficiente de mortalidade materna no municipio de Osasco, S. Paulo, Brasil. Rev. Saúde puibl., S. Paulo, 6: 237-44, 1972.

\section{RSPSP-136}

CinRt JR., C. \& Almeida, P.A.M. de - [Analysis of maternal mortality rate in the county of Osasco, S. Paulo State, Brazil]. Rev. Saúde públ., S. Paulo, 6: $237-44,1972$.

Summary: The maternal mortality rate in the county of Osasco (S. Paulo State, Brazil) was analysed and it was observed that this rate is still high comparing with the city of S. Paulo (S. Paulo State, Brazil), as well as with towns of more developed countries. Even so, one can notice that this rate shows a real improvement in the maternal health service with a reduction of the rate of $28,99 \%$ during the 1967-1970 period. The necessity that the maternal mortality rate be analized in respect to not only its magnitude but also as to its conduct during the different years, was showed. It is important to compare the obstetrical causes of death with others in the feminine fertile group and the relationship between the different obstetrical causes. The importance between these and the maternal health services, was showed.

UnITERMs: Maternal health*; Maternal mortality*; Prenatal care*.

\section{REFEREN,CIAS BIBLIOGRAFICAS}

1. ALONSO, J. - Estimativas populacionais e construgão de tabua de sobrevivencia para o sexo feminino no Municipio de Osasco. Osasco, Secretaria da Saúde. Departamento Técnico Normativo, 1971.

2. MANUAL de classificação estatística inter. nacional de doenças, lesóes e causas de óbito; 8.a revisão, 1965. Washington, D. C., Organização Panamericana da Saúde, 1969. (OPAS - Publicaçăo cientifica, 1900).

3. MONETTI, V. et al. - Situação da sậide materna $\theta$ infantil no Estado de São Paulo. São Paulo, Instituto de Saúde. Divisão de Saúde Materna e da Criança, 1970. (Publicą̧ão, 4 - Série D, n.॰ 1).

4. NOTICIARIO ESTATISTICO (Departamento de Estatística do Estado de s. Pau 10) São Paulo, (1):3-10, 1971.

5. PUFFER, R. R. \& GRIFFITH, G. W. Caracteristicas de la mortalidad urbana. Washington, D. C., Organización Panamericana de la Salud, 1968. p. 191_205 (Publiación cientifica, 161).

\footnotetext{
Recebido para publicacão em 17-4-1972

Aprovado para publicagão em 26-7-1972.
} 